\title{
Effects of Vestibular Training on Postural Control of Healthy Adults
}

Kwadwo O. Appiah-Kubi, PT, MPT, PhD¹, Anne Galgon, PT, MPT, $\mathrm{PhD}^{2}$, Ryan Tierney, ATC, PhD ${ }^{1}$, Richard Lauer, $\mathrm{PhD}^{1}$, W. Geoffrey Wright, $\mathrm{PhD}^{1}$

${ }^{1}$ Department of Health and Rehabilitation Sciences, Temple University College of Public Health

${ }^{2}$ Department of Physical Therapy, University of the Sciences

Correspondence: appiah@temple.edu (Kwadwo O. Appiah-Kubi)

\section{Background}

Vestibular dysfunction is one of the common causes of postural instability. About 70 million US adults aged $>40$ years experienced vestibular dysfunction and it has an annual economic cost of $\$ 16,550 /$ patient. ${ }^{1,2}$ Postural stability is essential in activities of daily living and it relies on the integration of multisensory inputs to drive motor outputs. When visual and somatosensory input is available and reliable, this reduces the postural control system's reliance on the vestibular system. Despite this, vestibular loss can still cause severe postural dysfunction. ${ }^{3,4}$ Training one or more of the three sensory systems can alter sensory weighting and change postural behavior. Vestibular activation exercises and weight shift training (WST) influence vestibular-ocular and motor responses and have been showed to be effective in vestibular rehabilitation. ${ }^{5-10}$ However, some groups of patients may not show improvement, probably due to, the type of vestibular rehabilitation employed. ${ }^{5}$ No study has employed a concurrent vestibular activation and WST to realize a more effective rehabilitation method by positively influencing sensory reweighting mechanisms and vestibular reflexes, except our pilot study. ${ }^{10}$ Our novel training method was tested on a healthy population to assess its feasibility.

\section{Purpose}

To assess sensory reweighting of postural control processing and vestibular-ocular and -motor responses after concurrent vestibular activation with postural training. We hypothesize the novel training will significantly alter the pattern of sensory weighting by changing the ratio of visual, somatosensory and vestibular dependence needed to maintain postural stability, and significantly decrease vestibular responses (vestibulo-ocular reflex [VOR], vestibulo-colic reflex [VCR] and vestibulo-spinal reflex [VSR]). 


\section{Methods}

Forty-two young healthy individuals (22 females, 20 males; $23.0+3.9$ years; $1.6+0.1$ meters; $67.5+15.2 \mathrm{~kg}$ ) were randomly assigned into four groups: 1) real time visual feedback weight shift training (WST) coupled with an active horizontal headshake (HHS), 2) same WST with vertical headshake (VHS), 3) WST with no headshake (NHS) and 4) no training or headshake (control, CTL) groups. The headshake groups performed an intensive body WST together with horizontal or vertical rhythmic headshake at 80 to 120 beats/minute. The NHS group performed the WST with no headshake while the controls did not perform any training. Five 15-minute training sessions were performed on consecutive days for one week with the weight shift exercises involving upright limits of stability activities on a flat surface, foam or rocker board (Figure 1).

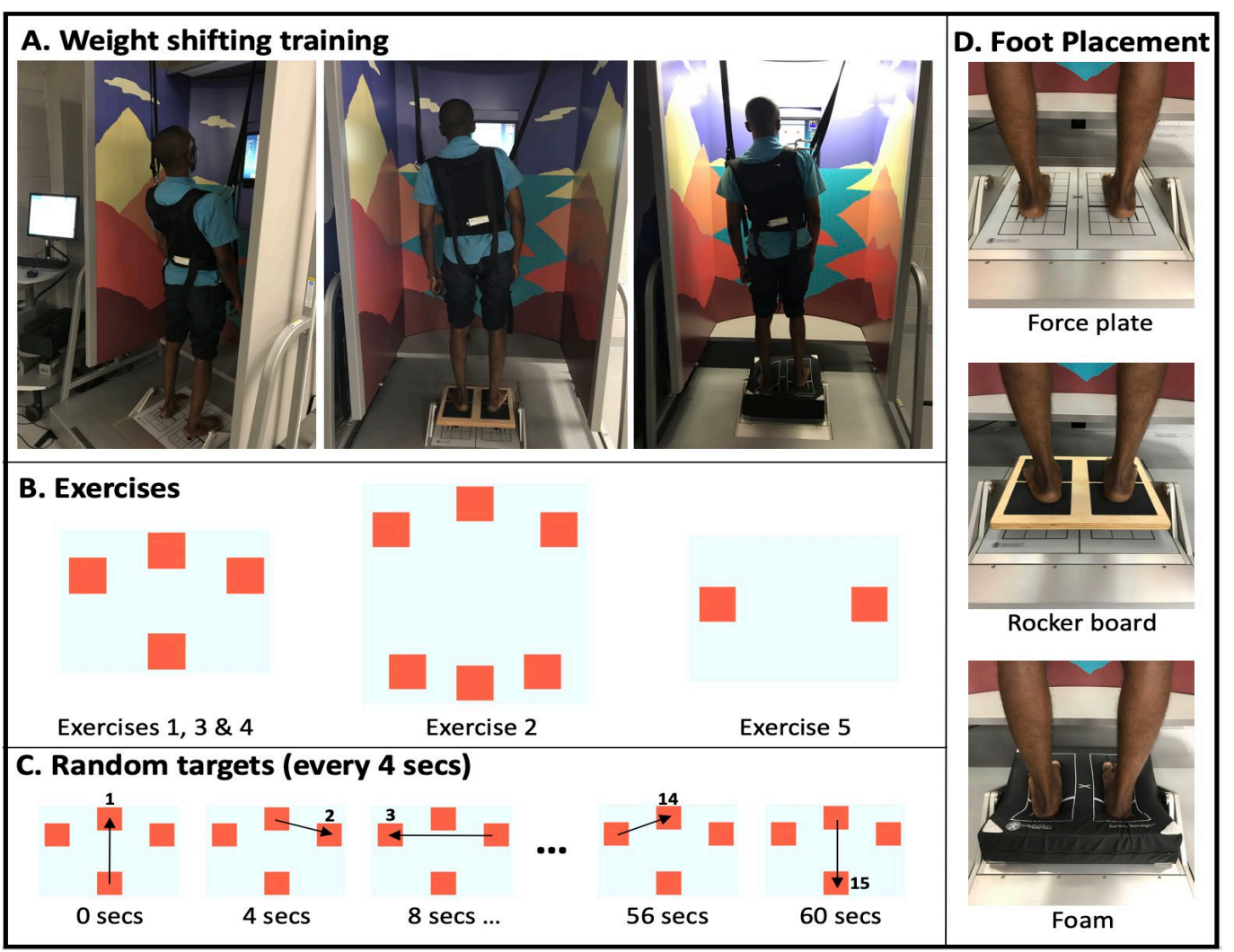

Figure 1. Training protocol. A. Weight shift exercises on force plate, rocker board and foam, with participant following directions on the screen; B-C. Screen interface showing visual feedback exercise targets of limits of stability (LOS), which changes randomly every 4 seconds; D. Foot placement during exercises

All groups performed baseline- and post-assessments including sensory organization test $(\mathrm{SOT})^{11,12}$ and force platform ramp perturbations, coupled with electromyographic (EMG) recordings. A video head impulse test was also used to record horizontal vestibulo-ocular reflex (VOR) gain. A between- and within-group repeated measures ANOVA was used to analyze five center of pressure (COP) sway variables, the equilibrium and composite scores and sensory ratios of the SOT as well as EMG signals and horizontal VOR gain. Similarly, COP variables, 
EMG, as well as vestibular reflex data (vertical VOR, VCR and VSR gains) during ramp perturbations were analyzed. Alpha level was set at $\mathrm{p}<.05$.

\section{Results}

The groups were not significantly different in age, gender, height, weight and body mass index. The training showed a significant somatosensory downweighting $(\mathrm{p}=.050)$ in the combined headshake groups compared to NHS and CTL groups. Similarly, when the headshake groups were pooled together, the training showed significant decreased horizontal VOR gain $(\mathrm{p}=.040)$ in the headshake groups. There was a faster automatic postural response (APR; $\mathrm{p}=.003$ ) (Figures $2-4)$ with improved flexibility $(p=.010)$ in the headshake groups.

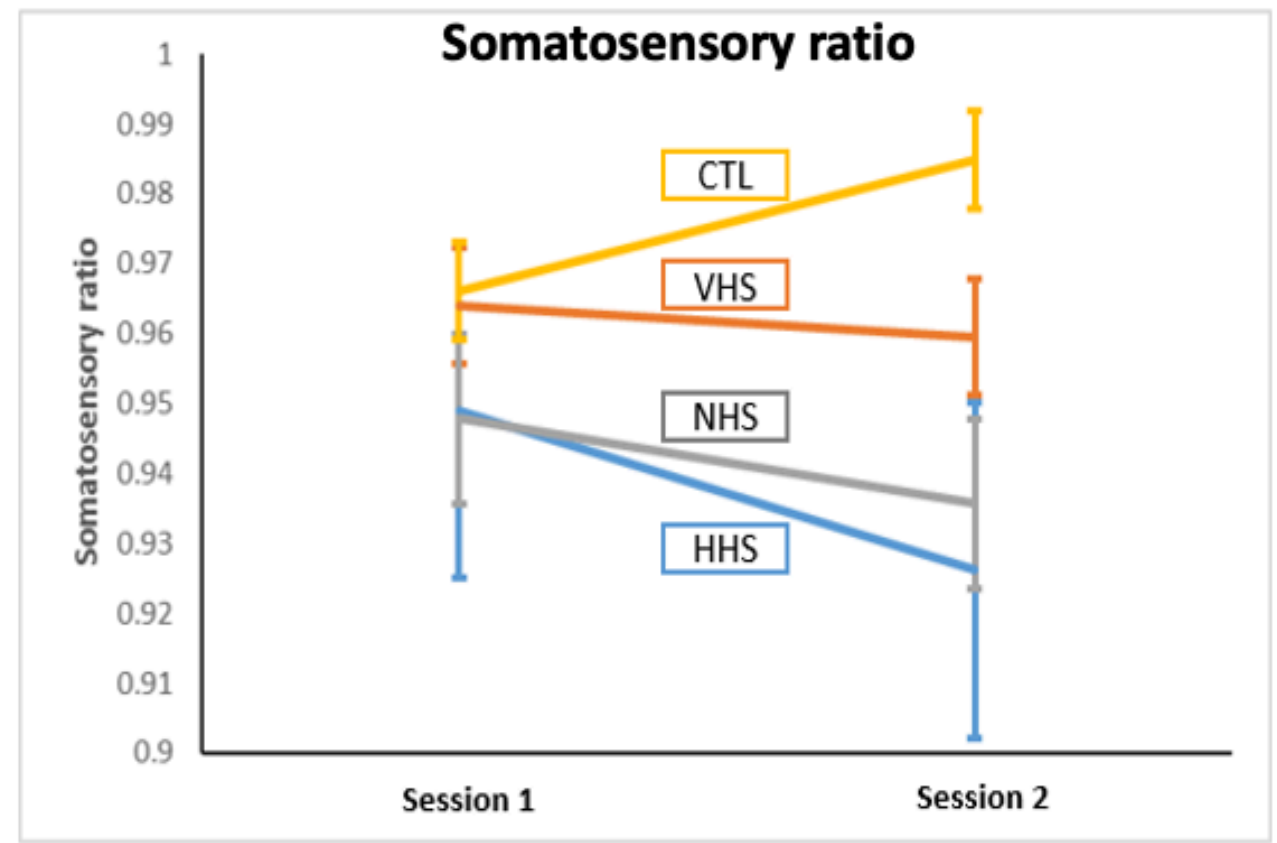

Figure 2. Somatosensory ratio. Somatosensory ratio was calculated as a ratio of SOT condition 2 to condition 1 . HHS = horizontal headshake; VHS = vertical headshake; NHS = no headshake; CTL = control; $\mathrm{p}=0.05$ when HHS and VHS as one group is compared with NHS and CTL 


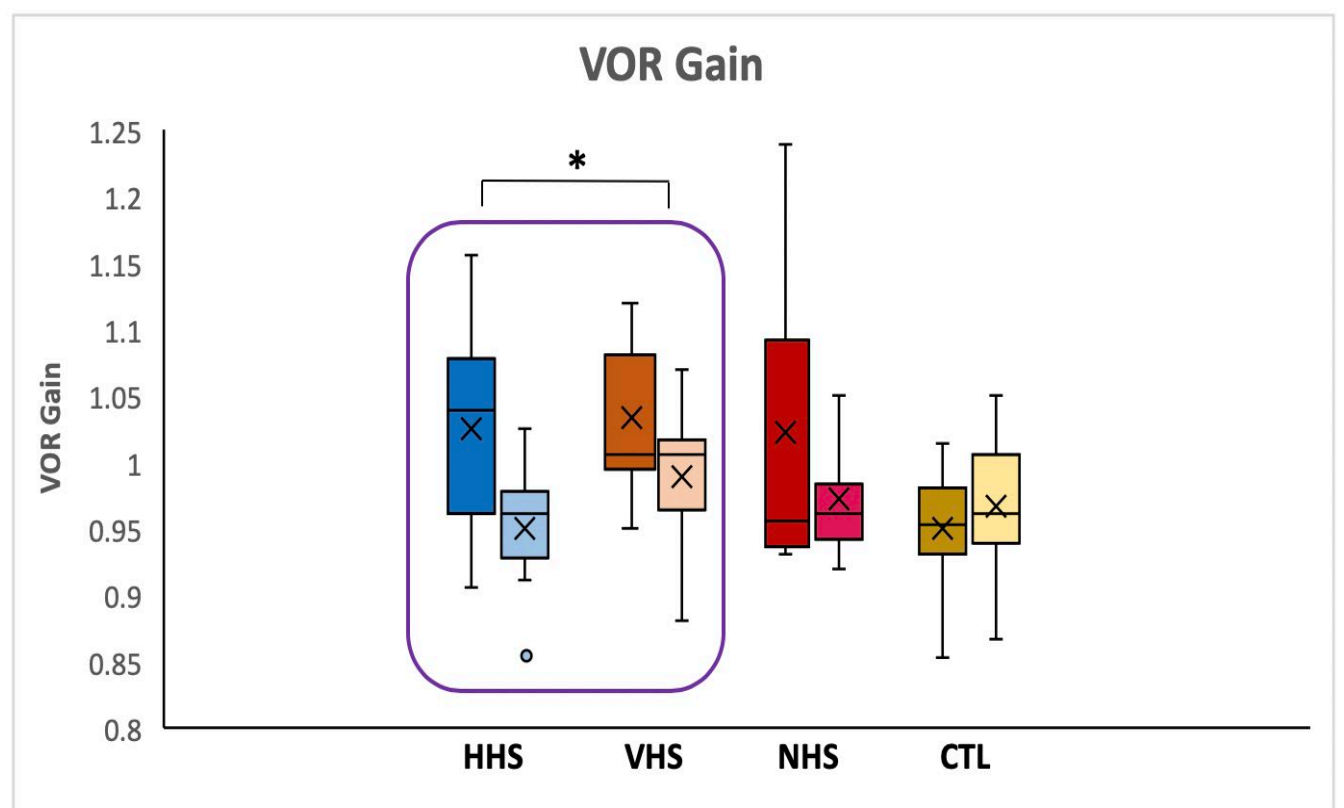

Figure 3. VOR gain for the 4 groups. Darker and lighter colors indicate assessment sessions 1 and 2, respectively. HHS = horizontal headshake; VHS = vertical headshake; NHS = no headshake; $\mathrm{CTL}=$ control; $\mathrm{p}<0.05$

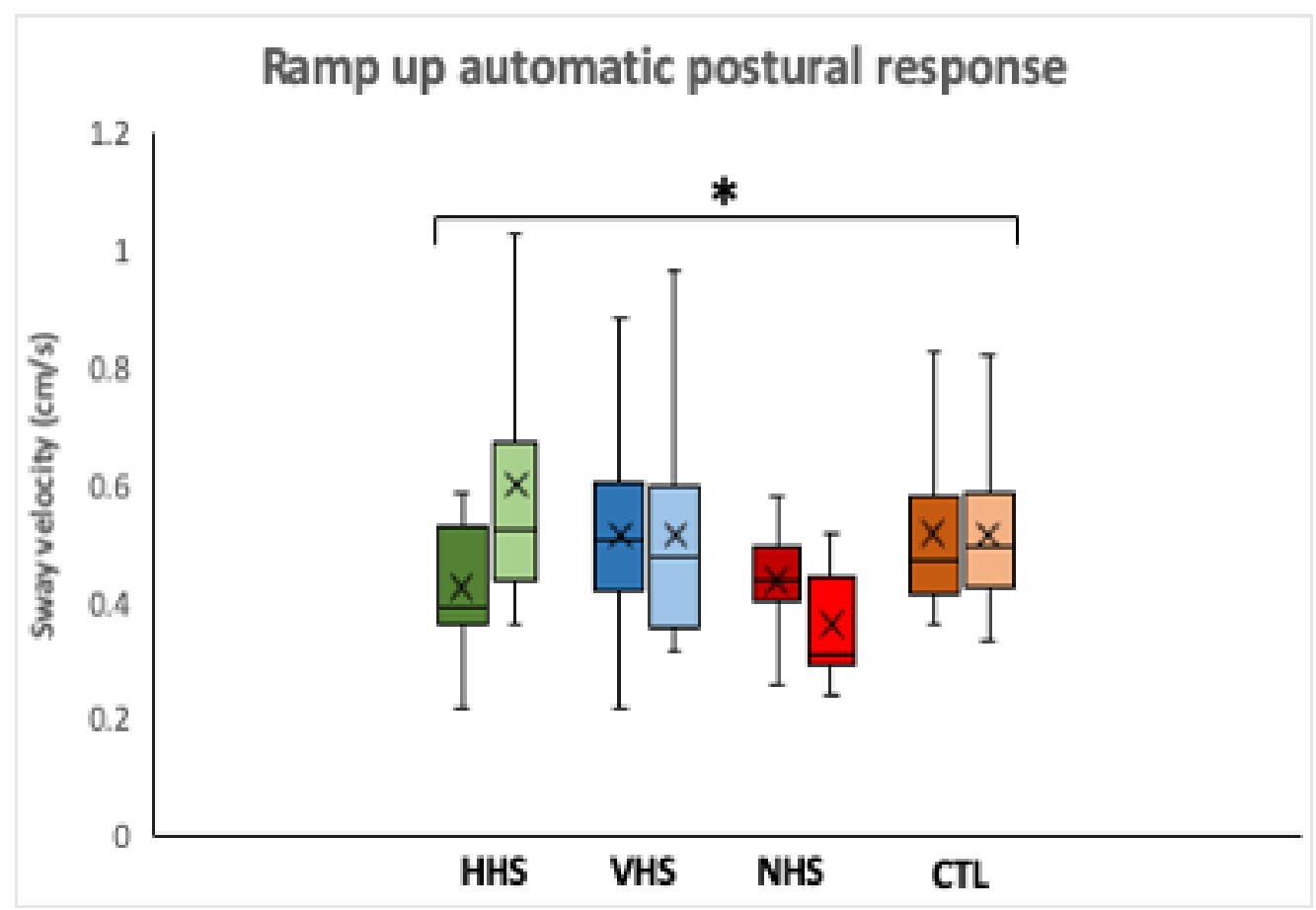

Figure 4. COP sway velocity (automatic postural response) for ramp up trials. Darker and lighter colors indicate assessment sessions 1 and 2, respectively. HHS = horizontal headshake; VHS = vertical headshake; NHS = no headshake; CTL $=$ control; $\mathrm{p}<0.05$ 
Muscle activation pattern in medial gastrocnemius $(\mathrm{p}=.033)$ was significantly decreased in the headshake during SOT conditions. ${ }^{4-6}$ The training, however, did not show significant differences in SOT equilibrium and composite scores, and vertical VOR, VCR and VSR gains.

\section{Conclusion}

The concurrent vestibular activation and weight shift training modifies some vestibulardependent responses after the training intervention as evidenced in somatosensory downweighting, decreased VOR gain, better postural flexibility and faster automatic postural response. Findings suggest this is predominantly due to vestibular adaptation and habituation of VOR, and partly vertical VOR, VCR and VSR which induced sensory reweighting in the headshake groups, as seen in the APR and flexibility. The benefits of the training protocol can also be translated to healthy athletes to optimize balance-related performance during sports activities.

\section{Clinical Relevance}

Findings may be used to guide the development of a vestibular-postural rehabilitation intervention in impaired neurological populations, such as with vestibular disorders or sensory integration problems present in traumatic brain injuries. Furthermore, the training protocol may be modified for home-based training with virtual reality goggles or using smart-phone solutions, which will increase portability, accessibility and affordability.

\section{Disclosures and Conflicts of Interest}

No conflicts of interest to report. This poster was presented at the 2019 Temple University College of Public Health Research Day.

\section{Funding sources}

This project was funded by a doctoral dissertation completion grant from Temple University.

\section{Acknowledgments}

The authors thanks Mark Jankowski and Christopher Haslam of Motion Action Perception Laboratory, Temple University, PA, USA. 


\section{References}

1. Agrawal Y, Carey JP, Della Santina CC, Schubert MC, Minor LB. Disorders of Balance and Vestibular Function in US Adults. Arch Intern Med [nternet]. 2009;169(10):938. Available from: http:/ / archinte.jamanetwork.com/article.aspx?doi=10.1001/archinternmed.2009.66

2. Sun DQ, Ward BK, Semenov YR, Carey JP, Della Santina CC. Bilateral vestibular deficiency: Quality of life and economic implications. JAMA Otolaryngol - Head Neck Surg. 2014;140(6):527-34.

3. Nashner L, Berthoz A. Visual contribution to rapid motor responses during postural control. Brain Res. 1978;150(2):403-7.

4. Nashner LM. Adaptation of human movement to altered environments. Trends Neurosci. 1982;5(C):358-61.

5. Herdman S. Vestibular rehabilitation. Curr Opin Neurol. 2013;26(1):96-101.

6. Lee YJ, Shin JE, Park MS, Kim JM, Na BR, Kim CH, et al. Comprehensive analysis of headshaking nystagmus in patients with vestibular neuritis. Audiol Neurotol. 2012;17(4):228-34.

7. Telian SA, Shepard NT, Smith-Wheelock M, Kemink JL. Habituation therapy for chronic vestibular dysfunction: Preliminary results. In: Otolaryngology - Head and Neck Surgery. 1990;103(1):89-95.

8. Shepard NT, Telian SA, Raj A, Smith-Wheelock M. Vestibular and balance rehabilitation therapy. Ann Otol Rhinol Laryngol. 1993;102(3):198-205.

9. Ricci N a, Aratani MC, Doná F, Macedo C, Caovilla HH, Ganança FF. A systematic review about the effects of the vestibular rehabilitation in middle-age and older adults. Rev Bras Fisioter. 2010;14(5):361-71.

10. Kwadwo O Appiah-Kubi, WG Wright. Vestibular training promotes adaptation of multisensory integration in postural control. Gait Posture. 2019;73:215-20.

11. Alsalaheen BA, Mucha A, Morris LO, Whitney SL, Furman JM, Camiolo-Reddy CE, et al. Vestibular rehabilitation for dizziness and balance disorders after concussion. J Neurol Phys Ther. 2010;34(2):87-93.

12. Mirka A, Black FO. Clinical application of dynamic posturography for evaluating sensory integration and vestibular dysfunction. Neurologic Clinics. 1990.

\section{Statement of Contributions}

Kwadwo O. Appiah-Kubi conceived the research idea, developed the theoretical framework and methodology, and conducted the entire study. Anne Galgon assisted in developing the training protocol and edited the manuscript. Ryan Tierney assisted in discussing portions of the framework and edited the manuscript. Richard Lauer assisted in formulating EMG aspects of the study and edited the manuscript. W. Geoffrey Wright assisted in developing the theoretical framework, methodology, discussion of results and edited the manuscript. 